Revue d'histoire du XIXe siècle

Société d'histoire de la révolution de 1848 et des

révolutions du XIXe siècle

23 | 2001

Nouvelles approches en histoire économique

\title{
Ernesto Mario BOLASCO, Damasco-Medina, ferrovia avveniristica (1901-1908). Mille operai italiani nel
} Deserto dell'Hedjaz

Quaderni di "Affari Sociali Internazionali", Milan, Franco Angeli, 1999, $116 \mathrm{p}$.

Nora Lafi

\section{OpenEdition}

Journals

Édition électronique

URL : http://journals.openedition.org/rh19/324

DOI : $10.4000 /$ rh 19.324

ISSN : $1777-5329$

Éditeur

La Société de 1848

Édition imprimée

Date de publication : 1 décembre 2001

Pagination : 237-239

ISSN : 1265-1354

Référence électronique

Nora Lafi, «Ernesto Mario BOLASCO, Damasco-Medina, ferrovia avveniristica (1901-1908). Mille operai italiani nel Deserto dell'Hedjaz », Revue d'histoire du XIXe siècle [En ligne], 23 | 2001, mis en ligne le 15 octobre 2002, consulté le 22 septembre 2020. URL : http://journals.openedition.org/rh19/324 ; DOI : https://doi.org/10.4000/rh19.324

Ce document a été généré automatiquement le 22 septembre 2020.

Tous droits réservés 


\section{Ernesto Mario BOLASCO, Damasco-} Medina, ferrovia avveniristica

\section{(1901-1908). Mille operai italiani nel Deserto dell'Hedjaz}

Quaderni di "Affari Sociali Internazionali", Milan, Franco Angeli, 1999, $116 \mathrm{p}$.

Nora Lafi

Ernesto Mario Bolasco, journaliste, puis diplomate, membre de la Commission italienne pour la publication des documents diplomatiques, livre dans la collection d'histoire diplomatique de l'éditeur Franco Angeli une belle contribution sur l'histoire de la construction de la ligne ferroviaire Damas-Médine (1901-1908), qui dépasse le seul cadre de l'étude des relations internationales. L'histoire de la construction de cette ligne de chemin de fer est en effet l'occasion d'offrir un panorama à la fois de la modernisation de l'Empire ottoman, des influences européennes dans ce processus, et d'histoire sociale, au travers de l'étude du destin d'un groupe d'ouvriers italiens.

L'auteur fonde sa démarche de recherches sur la consultation des précieuses archives du Ministère des Affaires étrangères italien, et particulièrement sur un rapport du consul Carrara, envoyé sur place pour étudier tant les conditions de vie des citoyens italiens, que l'état d'avancement d'un chantier hautement stratégique.

Le projet de construction de cette ligne de chemin de fer a été conçu par le gouvernement du sultan ottoman Abdul Hamid II à la fin du XIX siècle, dans le cadre des réformes de l'Empire (Tanzimât). La longueur de la ligne du Hedjaz telle qu'initialement prévue était de $1750 \mathrm{~km}$. Le sultan entendait réduire le temps de voyage de centaines de milliers de pèlerins vers les lieux saints de la religion musulmane, et confirmer de la sorte son autorité religieuse de Calife de l'Islam. Le projet revêtait également d'importants aspects militaires, pour le contrôle d'une province à la fois instable et convoitée. Le gouvernement de la Porte avait aussi, mais dans une moindre mesure, des visées économiques dans la promotion du projet, notamment autour de la volonté d'étendre les zones mises en culture, et de réactiver le 
commerce sur une route d'antique tradition. Le but était d'ancrer la péninsule arabique dans l'aire d'influence économique impériale.

Confiées à des sociétés étrangères, la conception et la construction de la ligne débutèrent au tournant du siècle. Le tracé partait de Damas, où s'arrêtaient alors les lignes existantes (inaugurées en 1894), en provenance d'Alep et de Beyrouth. Le projet initial prévoyait la desserte de Médine, puis de la Mecque. Mais les 450 derniers kilomètres ne furent jamais construits, face à l'opposition des populations bédouines et au tarissement des financements. Le projet d'une branche supplémentaire pour relier la ligne à la mer Rouge fut de même abandonné, face cette fois à la farouche opposition d'une Grande Bretagne soucieuse de préserver l'exclusivité du canal de Suez sur cette route. Ces deux modifications firent perdre à la ligne ses deux principaux débouchés : le pèlerinage et la route des Indes. Il reste que le chantier fut une occasion de moderniser les structures de l'Empire dans sa marge arabique.

Les décisions en la matière furent placées d'emblée sous l'autorité d'une Commission centrale pour la ligne ferroviaire du Hedjaz, dont le sultan lui-même était président. La partie technique fut placée sous la tutelle de l'ingénieur allemand Heinrich Meissner, auquel le succès de la ligne de Bagdad avait valu le titre de Pacha. À Damas, une souscommission fut mise en place, pour la gestion quotidienne du chantier. Le coût total de l'opération était estimé à 32 millions de francs, sans compter les travaux d'excavation, confiés à l'armée. Le matériel fut acheté en Allemagne, essentiellement auprès de l'entreprise Kraus-Maffei de Munich. Le premier tronçon, de Damas à Maan, fut inauguré en 1904, le second, jusqu'à Médine, en 1908.

Bolasco s'attache à décrire en détail les conditions de vie et de travail des ouvriers italiens sur le chantier. Les Italiens, en effet, étaient, après les Turcs, les plus nombreux le long du chantier, pour assurer les tâches les plus ingrates de travaux de terrassement en plein désert. Entre insécurité, maladie, alcoolisme et conflits avec les employeurs, les travailleurs italiens étaient mis à rude épreuve, pour un salaire modique. L'auteur, grâce au rapport consulaire de Carrara, dont l'ouvrage propose de très beaux extraits, parvient à dresser un tableau original d'histoire sociale du travail en contexte ottoman. L'histoire de la main-d'œuvre immigrée européenne dans les pays musulmans n'avait pas été faite. La mission consulaire de Carrara, autour du péril de la peste, visait à obtenir, pour les Italiens, une amélioration des conditions d'hygiène et de vie. Elle fut en cela un succès.

Bolasco propose également une analyse intéressante, hélas trop courte et fondée sur trop peu d'archives, du rapport des populations bédouines de la péninsule à la tutelle ottomane. Le chantier fut en effet l'occasion, le prétexte et le moyen d'une confrontation avec des populations largement insoumises. Cette piste reste à étudier plus avant. Il en est de même pour la comparaison que l'auteur propose avec l'autre grand chantier de l'époque, dans un tout autre contexte : le canal de Panama. C'est là le défaut principal de l'ouvrage : une trop grande promptitude à ne faire qu'évoquer des arguments qui auraient pu chacun construire de beaux chapitres. La ligne de chemin fer d'Arabie, en effet, pouvait amener dans son étude, un regard nouveau sur l'histoire ottomane. Or le contexte ottoman est ici étudié avec trop peu d'attention pour donner à l'exemple toute sa résonance. Peut-être aurait-il fallu ne pas reprendre forcément les a priori sur "l'homme malade", et replacer le chantier et sa conception dans le cadre de l'effort de modernisation de l'Empire, sans en présager de l'échec. On ne sait pas en 1900 que la Première Guerre mondiale mettra à bas l'édifice impérial, et les efforts de modernisation doivent être analysés en tant que tels, quitte à 
en montrer les limites et les contradictions. Le contexte politique ottoman, autour notamment de l'épisode Jeunes Turcs, aurait pu de même être traité plus en détail, tout comme les prétentions d'autres nations sur la région. La lecture des aspects stratégiques de missions consulaires dans l'Empire à la veille de la guerre italoottomane aurait pu grandement bénéficier d'une attention plus poussée aux aspects politiques de l'action d'un personnage comme Carrara. Quant aux aspects économiques, on aurait aimé connaître mieux le détail des appels d'offres, ainsi que le détail sur les procédures d'embauche de la main d'œuvre immigrée.

Il reste que la contribution de Bolasco amène à la connaissance de la présence ottomane en Arabie au tournant des $\mathrm{XIX}^{\mathrm{e}}$ et $\mathrm{XX}^{\mathrm{e}}$ siècles une pierre inédite et originale, et qu'elle permet une stimulante relecture de nombreuses problématiques ayant trait tant au jeu des grandes puissances autour des provinces ottomanes que des dynamiques internes de modernisation de l'Empire. 